\title{
Mutant calreticulin causes essential thrombocythemia
}

\section{Kazuya Shimoda, Kotaro Shide and Takuro Kameda}

Polycythemia vera (PV), essential thrombocythemia (ET), and primary myelofibrosis (PMF) are categorized as myeloproliferative neoplasms (MPNs). All are characterized by the autonomous growth of one or more lineages of hematopoietic cells, splenomegaly, constitutional symptoms such as fatigue, night sweating, itching, and weight loss, and is frequently complicated by thrombosis and hemorrhage. $J A K 2$ mutations are observed in more than $95 \%$ of PV patients, and about half of ET and PMF patients. About two-thirds of JAK2 mutationnegative ET and PMF patients harbor Calreticulin (CALR) mutations. CALR mutations with a 52-bp deletion or a 5-bp insertion in exon 9 occur in more than $80 \%$ of patients with CALR mutations, and cause frameshifts that result in proteins with novel $\mathrm{C}$-terminal domains composed of many positively charged amino acids [1]. As $C A L R$ mutations are exclusively observed in conjunction with $J A K 2$ mutations in MPN patients, mutant CALR is speculated to have a driver role in MPNs.

Cytokines such as erythropoietin (EPO), thrombopoietin (TPO), and granulocyte colonystimulating factor bind to their specific cell surface receptors, commonly activate the JAK-STAT signaling cascade, and induce cytokine-dependent transient hematopoiesis [2]. In cases of JAK2 mutation, JAK2 and STAT5 are constitutively activated without cytokine stimulation, and cause autonomous cell growth (Figure 1). As CALR is best known for its endoplasmic reticulum chaperone functions, assisting in glycoprotein folding, the question has been raised as to why mutant CLAR causes MPN.

We recently reported that in the presence of MPL, mutant CALR augmented the transcriptional activity of STAT5, but not of EPO receptor or CSF3R [3]. When $C A L R$ mutations are knocked-in human hematopoietic cell lines expressing MPL using the CRISPR-Cas9 system, these cell lines demonstrate increased growth or acquire cytokine-independent cell growth accompanied by STAT5 phosphorylation. These observations indicate that mutant CALR induces cytokine-independent activation of the JAK-STAT signaling cascade in cells expressing MPL, which results in increased cell growth or cytokineindependent cell growth (Figure 1).

In line with these observations, CALR mutant mice demonstrate thrombocythemia and develop ET, but do not exhibit erythrocytosis or granulocytosis [3]. This is consistent with many reports in which ET patients with a CALR mutation have lower Hb levels and/or leukocyte counts compared to ET patients with a JAK2 mutation. In addition, ruxolitinib, which is a JAK inhibitor that ameliorates splenomegaly and constitutional symptoms associated with MF, and ameliorates elevated hematocrit values and splenomegaly associated with PV, attenuates the increased numbers of peripheral blood platelets and BM megakaryocytes in CALR mutant mice. The effect of ruxolitinib on mice with $C A L R$ mutations also supports the idea that mutant CALR autonomously activates the JAK-STAT signaling cascade and induces sustained thrombocytosis with increased numbers of megakaryocytes. Our observations, together with previous reports [4-7], indicate that the CALR mutation is sufficient to augment megakaryocytic cell growth and cause ET, and that CALR mutations, like the $J A K 2$ mutation, play a driver role in MPNs.

MPL is expressed not only on megakaryocytes, but also on hematopoietic stem cells (HSCs). TPO stimulation is reported to increase HSC numbers in vitro and in vivo. The proportion of HSCs in BM is higher in CALR mutant mice than in WT mice, which is probably due to the constitutional activation of STAT5 by mutant CALR in HSCs. Although the number of HSCs in BM from CALR mutant mice is elevated, HSCs with CALR mutation do not demonstrate greater self-renewal activity than WT HSCs. In the first recipients in serial competitive transplantation assays, BM cells with a CALR mutation exhibit a growth advantage compared to WT cells. In the second recipients, BM cells with a CALR mutation exhibit almost the same growth as WT BM cells. This also occurs in JAK2

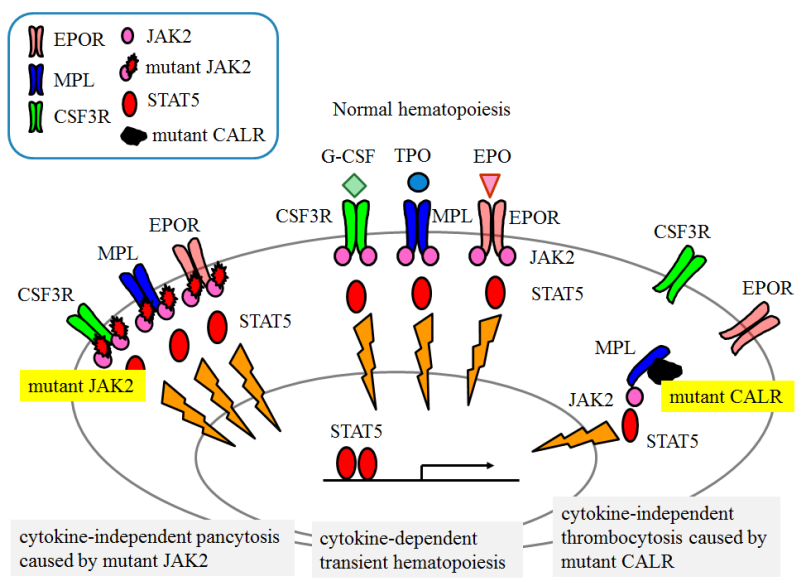

Figure 1: JAK-STAT signaling cascade in normal and MPN hematopoiesis. 
mutated-HSCs, suggesting that other kinds of mutations in addition to those of $C A L R$ and $J A K 2$ may be required for the full development of MPNs [8].

Mutant CALR plays a driver role in MPNs, however the precise mechanism whereby mutant CALR activates STAT5 has not been fully clarified. Araki et al. reported that mutant CALR, but not WT CALR, binds to MPL, and that the novel C-terminal domains of the mutant protein are required for this interaction [6]. Elf et al. reported that the transforming activity of mutant CALR is not contained within specific residues within the mutant CALR C-terminus, but the oncogenicity of this mutant is dependent on the positive electrostatic charge of its C-terminus [7]. We generated a murine CALR mutation knock-in mice via the deletion of base pairs in the murine CALR C-terminal (submitted manuscript, Shide K et al.). This murine mutant CALR possesses many positively charged amino acids, similar to the human mutant CALR, but the precise amino acid sequences are different from their human counterpart. Murine CALR mutation knockin mice do not develop ET, which suggests that newly generated amino acids in human CALR may be essential in the development of ET.

Kazuya Shimoda: Department of Gastroenterology and Hematology, Faculty of Medicine, University of Miyazaki, Miyazaki, Japan

Correspondence to: Kazuya Shimoda, email kshimoda@ med.miyazaki-u.ac.jp

Keywords: CALR, JAK2

Received: August 29, 2017

Published: September 28, 2017

\section{REFERENCES}

1. Klampfl T, et al. N Eng J Med. 2013; 369:2379-2390.

2. Ihle JN, et al. Stem Cells. 1997; 15:105-111.

3. Shide K, et al. Leukemia. 2017; 31:1136-1144.

4. Marty C, et al. Blood. 2016; 127:1317-1324.

5. Chachoua I, et al. Blood. 2016; 127:1325-1335.

6. Araki M, et al. Blood. 2016; 127:1307-1316.

7. Elf S, et al. Cancer Discovery. 2016; 6:368-381.

8. Kameda T, et al. Blood. 2015; 125:304-315.

Copyright: Shimoda et al. This is an open-access article distributed under the terms of the Creative Commons Attribution License 3.0 (CC BY 3.0), which permits unrestricted use, distribution, and reproduction in any medium, provided the original author and source are credited. 